\title{
ESTIMATES OF PLANAR REGIONS OF ASYMPTOTIC STABILITY*
}

\author{
BY LARRY R. ANDERSON (Whitman College, Walla Walla)
}

1. Introduction. In an earlier paper, the current author and Walter Leighton showed that in many general cases one could find weight functions $\theta(x, y)$ such that functions

$$
V_{\theta}=\int_{h(x)}^{y} \theta(x, u) f(x, u) d u-\int_{0}^{x} \theta(u, h(u)) g(u, h(u)) d u
$$

are Liapunov functions for a differential system $\dot{x}=f(x, y), \dot{y}=g(x, y)$, where $f(x, h(x)) \equiv$ 0 .

In the case of an isolated, asymptotically stable equilibrium point, an unsolved problem is the problem of finding a function $\theta$ so that the approximation by $V_{\theta}$ of the region of asymptotic stability is in some sense maximal among admissible values of $\theta$.

It was shown in Theorem 3.1 of [1] that in a large class of cases the estimate of the region of asymptotic stability given by the curves $V_{\theta}=c$ could always be improved. The current author was able to show that under the hypotheses of Theorem 3.1 of [1], one can find a function $g(x)$ and a number $\epsilon>0$ such that if $r(x, y, \epsilon)=p(x, y)+\epsilon g(x)$, then the estimate of the region of asymptotic stability given by $V_{r}$ totally contains the estimate given by $V_{p}$. The argument is similar to that given in [1, pp 662-663] and is omitted here.

The above suggests that in at least a large number of cases there is no "best" choice of $\theta$. Given this, is it possible to consider some subclass of admissible functions and to maximize estimates over this subclass? Can one take an estimate given by $V_{\theta}$ and substantially increase the estimate with a function $V_{\alpha}$ ?

In what follows, it will be seen that in a special case the answer to the above questions is in the affirmative. Below, we take $V$ to be $V_{\theta}$, where $\theta=x^{2} a^{-2}+y^{2} b^{-2}$, and obtain an estimate that improves an estimate in [4] that is derived by considering level curves of $\left(2 V_{\theta}\right)^{1 / 2}, \theta=x^{2}+y^{2}$. (In the system considered below, the roles of $f, g, x, y$ are reversed in the construction of $V_{\theta}=V$.)

2. An estimate for the van der Pol equation. It is well known that the system

$$
\begin{aligned}
& \dot{x}=y-\epsilon\left(\frac{x^{3}}{3}-x\right) \quad(\epsilon<0), \\
& \dot{y}=-x
\end{aligned}
$$

has a unique limit cycle $\Omega$. In this section we give an estimate for the diameter of this limit cycle. An estimate of $2 \sqrt{ } 3$ is developed in [4], and in the following we improve that estimate.

* Received November 11, 1977: revised version received January 30, 1978. The author would like to thank Prof. T. Lindstrom for preparing the numerical data used in sketching the curves in Sec. 3. 
We define

$$
V(x, y)=\frac{x^{2} y^{2}}{2 b^{2}}+\frac{y^{4}}{4 b^{2}}+\frac{x^{4}}{4 a^{2}}
$$

where $a$ and $b$ are positive constants to be determined. We shall assume throughout the sequel that $0<a<1$.

A little computation shows that

$$
\dot{V}=x^{2} \epsilon\left[\frac{y^{2}}{b^{2}}+\left(\frac{1}{a^{2} \epsilon}-\frac{1}{b^{2} \epsilon}\right) x y+\frac{(1-a)}{a^{2}} x^{2}+x^{2}\left(\frac{1}{a}-\frac{x^{2}}{3 a^{2}}-\frac{y^{2}}{3 b^{2}}\right)\right],
$$

where $\dot{V}$ is the derivative of $V$ taken along trajectories of the system (1).

For $x \neq 0, \dot{V}$ will be negative inside the ellipse

$$
\frac{1}{a}=\frac{x^{2}}{3 a^{2}}+\frac{y^{2}}{3 b^{2}}
$$

if the quadratic form

$$
\frac{y^{2}}{b^{2}}+\left(\frac{1}{a^{2} \epsilon}-\frac{1}{b^{2} \epsilon}\right) x y+\frac{(1-a) x^{2}}{a^{2}}
$$

is positive semidefinite-that is, if

$$
\frac{1}{\epsilon^{2}}\left(\frac{b^{2}-a^{2}}{a^{2} b^{2}}\right)^{2}-\frac{4(1-a)}{a^{2} b^{2}} \leq 0 .
$$

This inequality is equivalent to the inequality $\left|b^{2}-a^{2}\right| \leq 2|\epsilon| a b(1-a)^{1 / 2}$. We assume henceforth that $b>a$, and the latter inequality reduces to

$$
0<\frac{b}{a}-\frac{a}{b} \leq 2(1-a)^{1 / 2}|\epsilon| .
$$

Now the curves $V=c(c>0)$ will bound a region of stability of the origin when (4) holds and when the curves $V=c$ lie inside the region defined by (3), i.e. inside the region

$$
x^{2}=3 a^{2}\left(\frac{1}{a}-\frac{y^{2}}{3 b^{2}}\right)=3 a-\frac{y^{2} a^{2}}{b^{2}} .
$$

We can solve the equation $V=c$ for $x^{2}$. We obtain

$$
x^{2}=\frac{-y^{2} a^{2}}{b^{2}}+\frac{a^{2}}{b^{2}}\left(y^{4}\left(1-\frac{b^{2}}{a^{2}}\right)+\frac{4 c b^{4}}{a^{2}}\right)^{1 / 2} .
$$

The region enclosed by (5) contains the region enclosed by (6) if

$$
3 a-\frac{y^{2} a^{2}}{b^{2}} \geq \frac{-y^{2} a^{2}}{b^{2}}+\frac{a^{2}}{b^{2}}\left(y^{4}\left(1-\frac{b^{2}}{a^{2}}\right)+\frac{4 c b^{4}}{a^{2}}\right)^{1 / 2},
$$

which reduces to $b^{4}(9-4 c) \geq 0$.

Accordingly, the curve $V=9 / 4$ contains a region of stability of the origin if condition (4) holds. It is well known that any such region must lie inside $\Omega$, and hence the curve $V=$ $9 / 4$ lies inside $\Omega$ if (4) holds. (One could also show that the set $\{X \mid \dot{V}(X)=0\}$ contains no invariant set other than the origin and hence $V=9 / 4$ encloses a region of asymptotic stability when condition (4) holds.) 
We note that the curve $V=c$ is symmetric in the origin. Thus a lower estimate for the diameter of $\Omega$ is obtained by taking twice the distance between the origin and any point on the curve $V=c$. The square of the distance from any point on $V=c$ to the origin can be computed from (6). This squared distance is

$$
y^{2}\left(1-\frac{a^{2}}{b^{2}}\right)+\frac{a^{2}}{b^{2}}\left(y^{4}\left(1-\frac{b^{2}}{a^{2}}\right)+\frac{4 c b^{4}}{a^{2}}\right)^{1 / 2} .
$$

If we put

$$
P(y)=y^{2}\left(1-\frac{a^{2}}{b^{2}}\right)+\frac{a^{2}}{b^{2}}\left(y^{4}\left(1-\frac{b^{2}}{a^{2}}\right)+\frac{4 c b^{4}}{a^{2}}\right)^{1 / 2} \quad\left(0 \leq y \leq\left(4 b^{2} c\right)^{1 / 4}\right),
$$

then one can show that the maximum value of $P$ occurs at the end point $y=\left(4 b^{2} c\right)^{1 / 4}$. Thus, with our function $V$, an optimal estimate of the diameter of $\Omega$ is given by $2\left(4 b^{2} c\right)^{1 / 4}$, which becomes $2(3 b)^{1 / 2}$ when $c=9 / 4$.

We now determine $b$ so as to maximize $2(3 b)^{1 / 2}$ above. First, let $a \in(0,1)$ be fixed. The function $r(x)=x a^{-1}-a x^{-1}(a \leq x)$ is strictly increasing on $[a, \infty)$, and hence the largest value of $b$ satisfying (4) is given by $b a^{-1}-a b^{-1}=2(1-a)^{1 / 2}|\epsilon|$, which reduces to $b^{2}-$ $2(1-\mathrm{a})^{1 / 2} a b|\epsilon|-a^{2}=0$. Solving, we obtain $b=a\left(|\epsilon|(1-a)^{1 / 2}+\left(\epsilon^{2}(1-a)+1\right)^{1 / 2}\right)$. Next, we find the largest value of the above expression for $a \in(0,1)$. Put $F(a)=a(|\epsilon|(1$ $\left.-a)^{1 / 2}+\left(\epsilon^{2}(1-a)+1\right)^{1 / 2}\right), 0<a<1$, and let $a=1-t$. We obtain $\gamma(t)=F(1-t)=|\epsilon|$ $\left(t^{1 / 2}+\left(t+\epsilon^{-2}\right)^{1 / 2}\right)(1-t)$. Note that $\lim _{t \rightarrow 0}+\gamma(t)=1$ and that $\gamma(1)=0$. Now

$$
\gamma^{\prime}(t)=|\epsilon|\left[\frac{(1-3 t)}{2 t^{1 / 2}}+\frac{1}{2\left(t+\frac{1}{\epsilon^{2}}\right)^{1 / 2}}\left(1-3 t-\frac{2}{\epsilon^{2}}\right)\right]
$$

for $0<t<1$. Since $\lim _{t \rightarrow 0^{+}} \gamma^{\prime}(t)=+\infty$, it follows that the maximum value of $\gamma$ on $[0,1]$ occurs in $(0,1)$. Moreover, this maximum value is greater than one.

The only critical point of $\gamma$ in $(0,1)$ occurs at

$$
t_{0}=\left[-\left(2+\frac{4}{\epsilon^{2}}\right)+\left(\left(2+\frac{4}{\epsilon^{2}}\right)^{2}+12\right)^{1 / 2}\right] / 6
$$

and thus $\gamma\left(t_{0}\right)$ is the maximum value of $\gamma$ on $[0,1]$. The maximum value of $F$ then occurs at $a_{0}=1-t_{0}$. Letting

$$
a_{0}=1+\left[\left(2+\frac{4}{\epsilon^{2}}\right)-\left(\left(2+\frac{4}{\epsilon^{2}}\right)^{2}+12\right)^{1 / 2}\right] / 6
$$

and

$$
b_{0}=a_{0}\left(|\epsilon|\left(1-a_{0}\right)^{1 / 2}+\left(\epsilon^{2}\left(1-a_{0}\right)+1\right)^{1 / 2}\right),
$$

we obtain the following result.

THEOREM: For all $\epsilon<0$, the curve

$$
\frac{x^{2} y^{2}}{2 b_{0}^{2}}+\frac{y^{4}}{4 b_{0}^{2}}+\frac{x^{4}}{4 a_{0}^{2}}=\frac{9}{4}
$$

bounds a region of asymptotic stability of the origin for the system (1). The diameter of this region is a function $G$ of $\epsilon$ given by

$$
G(\epsilon)=2 \sqrt{3} \cdot \sqrt{a}_{0}\left(|\epsilon|\left(1-a_{0}\right)^{1 / 2}+\left(\epsilon^{2}\left(1-a_{0}\right)+1\right)^{1 / 2}\right)^{1 / 2}
$$




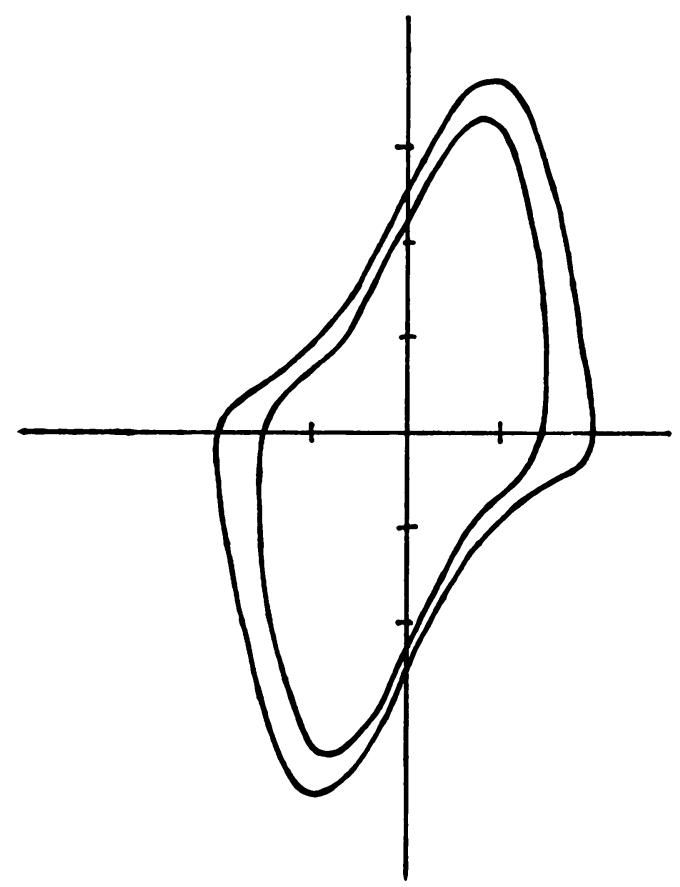

Fig. 1. Outer curve is the periodic orbit for Eq. (9) and the inner curve is $(8), \epsilon=-2$.

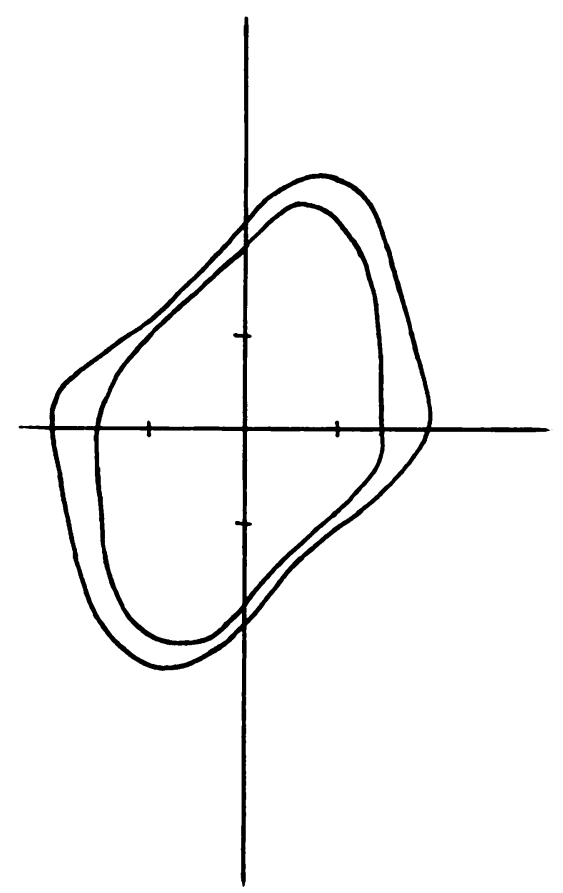

FIG. 2. Outer curve is the periodic orbit for Ea. (9) and the inner curve is (8), $\epsilon=-1$. 
and satisfies

$$
\begin{aligned}
& \text { (a) } G(\epsilon)>2 \sqrt{3} \text { for all } \epsilon<0, \\
& \text { (b) } \lim _{|\epsilon| \rightarrow \infty} \frac{G(\epsilon)}{\sqrt{|\epsilon|}}=\frac{4}{3^{1 / 4}} .
\end{aligned}
$$

It is clear that the above estimate generalizes the estimate $2 \sqrt{3}$ given in [4]. Moreover, we have derived explicitly a set of curves that approximate the region within $\Omega$. These curves enlarge as $|\epsilon|$ grows, but the rate of growth is not sharp-i.e., the estimate obtained for the diameter $D(\epsilon)$ of $\Omega$ is not good for $|\epsilon|$ large. Indeed, it is known (see [2]) that $D(\epsilon)$ satisfies $D(\epsilon) \sim 4 / 3|\epsilon|$ as $|\epsilon| \rightarrow \infty$.

In [3], the author provides a method for constructing an annular region containing $\Omega$ by constructing closed curves interior and exterior to $\Omega$. This region can be modified by using the curve in our theorem as the interior curve rather than the circle of fixed radius $\sqrt{3}$ given in [3].

Finally, we point out that the trajectories of the system (1) are the same as those of the van der Pol equation except that their orientation is reversed (see [4]).

3. An estimate for an equivalent system. The system of differential equations considered above is, of course, a system associated with the van der Pol equation

$$
\ddot{x}=\epsilon\left(1-x^{2}\right) \dot{x}-x \quad(\epsilon>0) \text {. }
$$

In the system considered in Sec. 2 we note that $\dot{x}=y-\epsilon\left(x^{3} / 3-x\right)$, where $x$ is a solution of (7). Then

$$
y=\dot{x}+\epsilon\left(\frac{x^{3}}{3}-x\right)
$$

and the curve in our theorem becomes

$$
\frac{9}{4}=\frac{x^{2}\left[\dot{x}+\epsilon\left(\frac{x^{3}}{3}-x\right)\right]^{2}}{2 b_{0}{ }^{2}}+\frac{\left[\dot{x}+\epsilon\left(\frac{x^{3}}{3}-x\right)\right]^{4}}{4{b_{0}{ }^{2}}^{4}}+\frac{x^{4}}{4{a_{0}}^{2}} .
$$

If we replace $\epsilon>0$ by $\epsilon<0$ (see [4]) in (7), the preceding curve then encloses a region of asymptotic stability for $(7)$ (with $\epsilon<0$ ) relative to the system

$$
\dot{x}=y, \quad \dot{y}=\epsilon\left(1-x^{2}\right) y-x,
$$

i.e., the phase space of (7).

The curve in (8) is illustrated in Figs. 1 and 2 in two separate cases. In each case the outer curve is the limit cycle for (9) and the inner curve is the curve (8).

\section{REFERENCES}

[1] L. Anderson and W. Leighton, Liapunov functions for autonomous systems of second order, J. Math. Anal. Appl. 23, 645-664 (1968)

[2] P. Brock, The nature of solution of a Rayleigh type forced vibration equation with a large coefficient of damping. J. Appl. Phys. 24, 1004-1007 (1953)

[3] R. J. P. de Figueiredo, Existence and uniqueness results for Liénard's equation, I.E.E.E. Trans. CT17, 313-321 (1970)

[4] J. P. LaSalle and S. Lefschetz, Stability by Liapunov's direct method, with applications, Academic Press, New York, 1961 